\title{
Left atrial appendage occlusion in a patient with hereditary hemorrhagic telangiectasia and atrial fibrillation - a therapeutic option worth considering
}

Maksymilian Mielczarek ${ }^{1}$, Rafał Gałąska ${ }^{1}$, Piotr Drewla ${ }^{1}$, Dorota Kulawiak-Gałąska², Dariusz Ciećwierz ${ }^{1}$, Marcin Gruchała ${ }^{1}$

${ }^{1} 1^{\text {st }}$ Department of Cardiology, Medical University of Gdansk, Gdansk, Poland ${ }^{2}$ Department of Radiology, Medical University of Gdansk, Gdansk, Poland

Submitted: 31 May 2017

Accepted: 12 June 2017

Arch Med Sci Atheroscler Dis 2017; 2: e29-e30

DOI: https://doi.org/10.5114/amsad.2017.68657

Copyright $\odot 2017$ Termedia \& Banach

Hereditary hemorrhagic telangiectasia $(\mathrm{HHT})$ is a dominantly inherited genetic disorder of blood vessel development characterized by epistaxis, mucocutaneous telangiectasias and arteriovenous malformations (AVMs). Recently, it was shown that HHT patients have a predilection to develop atrial fibrillation [1], which is probably due to frequent systemic AVMs, iron deficiency anemia and hypoxemia resulting in hyperdynamic circulation. Those patients cannot benefit from oral anticoagulant (OAC) therapy for stroke prevention, because of the unacceptably high bleeding risk [2]. Therefore left atrial appendage occlusion (LAAO) may be warranted in $\mathrm{HHT}$ patients with $\mathrm{AF}$ and high thromboembolic risk.

A 65-year-old woman with HHT was referred to our department for consideration of LAAO. The patient had developed gastric AVMs with several episodes of bleeding, which required repeated endoscopic treatment and blood transfusions. On the other hand, she had developed chronic AF with high thromboembolic risk according to $\mathrm{CHA}_{2} \mathrm{DS}_{2} \mathrm{VASC}$ (her score was 3, giving her an estimated annual risk of stroke of 3.2\%). The patient could not tolerate long-term OAC therapy. On that basis, LAAO was proposed by the Heart Team. Transesophageal echocardiography (TEE) and computed tomography angiography were performed to obtain measurements of the left atrial appendage (LAA) for occluder device sizing, as well as to exclude thrombus in the LAA (Figures $1 \mathrm{~A}, \mathrm{~B}$ ). The procedure was conducted under general anesthesia under TEE guidance. Femoral vein access was obtained. The interatrial septum was punctured with a dedicated needle and a transseptal sheath was inserted into the left atrium. Subsequently, a transseptal sheath was replaced over a stiff wire by a delivery sheath and an Amplatzer AMULET (St Jude Medical) occluder was deployed in the LAA (Figures 1 C-E). The patient was awakened and transferred to the Cardiac Intensive Care Unit for 24-hour surveillance. Recovery was uneventful and the patient was discharged on the $5^{\text {th }}$ day after LAAO. According to the standard protocol dual antiplatelet therapy was commenced, which consisted of aspirin $75 \mathrm{mg}$ daily and clopidogrel $75 \mathrm{mg}$ daily. Three months later TEE confirmed proper position of the device (Figure $1 \mathrm{~F}$ ). At that time clopidogrel was laid off. As low-dose aspirin therapy was well tolerated, the patient stayed with it.

According to European Society of Cardiology Guidelines, LAAO may be considered in patients with AF and contraindications for long-term OAC
Corresponding author:

Maksymilian Mielczarek MD, PhD

$1^{\text {st }}$ Department of Cardiology Medical University of Gdansk 7 Dębinki St 80-952 Gdansk, Poland Phone: +48583492504 E-mail: max.mielczarek@ gmail.com 


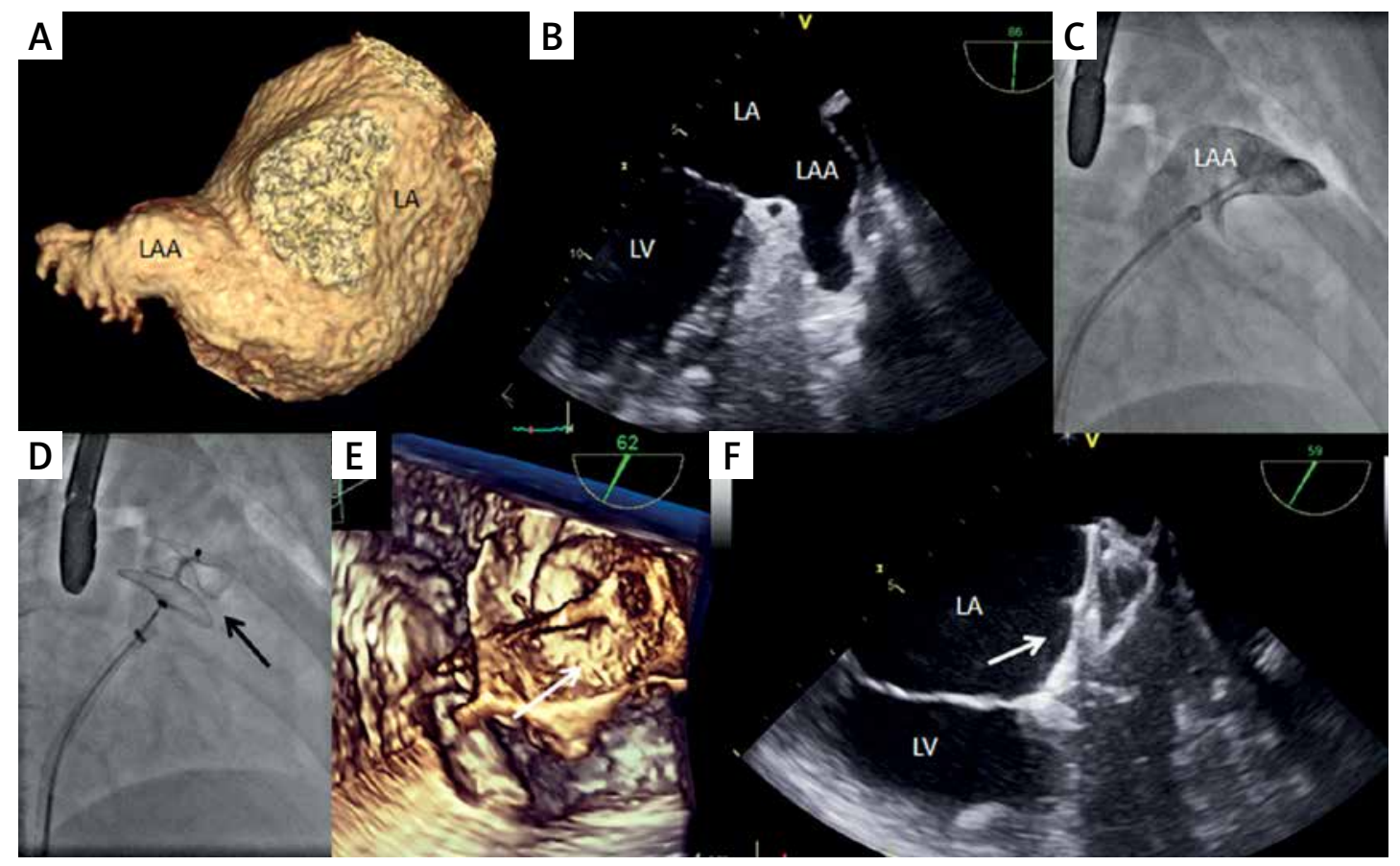

Figure 1. A - computed tomography angiography: 3D volume rendering of the left atrium (LA) and the left atrial appendage (LAA); $B$ - transesophageal echocardiography: 2D imaging of the left atrium (LA), the LAA and the left ventricle (LV); C - fluoroscopy: LAA angiography; D - fluoroscopy: Amplatzer AMULET (arrow) deployed in the left atrial appendage, but still attached to a delivery system; $\mathbf{E}$ - transesophageal echocardiography: 3D imaging of Amplatzer AMULET (arrow) deployed in the left atrial appendage, but still attached to a delivery system - en face view; $\mathbf{F}$ - transesophageal echocardiography: 2D imaging of the Amplatzer AMULET (arrow) implanted in the left atrial appendage - 3-month follow-up

therapy [3]. To date, only a few LAAO in HHT patients have been reported [4]. Due to the fact that $\mathrm{HHT}$ patients tolerate antiplatelet agents better than OAC [2], short-term dual antiplatelet therapy required after Amplatzer AMULET implantation can usually be completed.

\section{Conflict of interest}

The authors declare no conflict of interst.

\section{References}

1. Shovlin CL, Awan I, Cahilog Z, et al. Reported cardiac phenotypes in hereditary hemorrhagic telangiectasia emphasize burdens from arrhythmias, anemia and its treatments, but suggest reduced rates of myocardial infarction. Int J Cardiol 2016; 215: 179-85.

2. Devlin HL, Hosman AE, Shovlin CL. Antiplatelet and an ticoagulant agents in hereditary hemorrhagic telangiectasia. N Engl J Med 2013; 368: 876-8.

3. Kirchhof P, Benussi S, Kotecha D, et al. 2016 ESC Guidelines for the management of atrial fibrillation developed in collaboration with EACTS. Eur Heart J 2016; 37: 2893-962.

4. Vorselaars VM, Velthuis S, Swaans MJ, et al. Percutaneous left atrial appendage closure. An alternative strategy for anticoagulation in atrial fibrillation and hereditary hemorrhagic telangiectasia? Cardiovasc Diagn Ther 2015; 5: 49-53. 